ИЗВЕСТИЯ АКАДЕМИИ НАУК ЭСТОНСКОИ ССР. ФИЗИКА * МАТЕМАТИКА PROCEEDINGS OF THE ACADEMY OF SCIENCES OF THE ESTONIAN SSR. PHYSICS * MATHEMATICS

$1988,37,1$

УДК $532.783 ; 539.143 .43$

Р. ТЭЭЯЭР, Э. ЛИППМАА

\title{
ЯМР-ИССЛЕДОВАНИЕ ОРИЕНТАЦИИ ЖИДКОГО

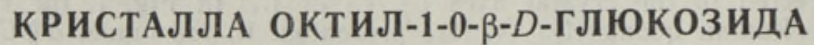 В МАГНИТНОМ ПОЛЕ
}

Причины синтеза в природе термодинамически невыгодной кристаллической структуры целлюлозы I неизвестны. Предприняты попытки объяснить особенности природного синтеза целлюлозы, исходя из гипотезы о реализации жидкокристаллического (ЖК) состояния на промежуточных его стадиях [ $\left.{ }^{1}\right]$. Согласно этой гипотезе, природные полимеры, возникающие в результате матричного синтеза, образуют на первой стадии метастабильные растворы, пересыщенные относительно окончательного (равновесного) состава фаз. Как правило, они способны кристаллизоваться, но возникновение зародышей кристаллических фаз - процесс сравнительно медленный, поскольку требует строгого взаимного расположения молекул. Значительно более быстрым является возникновение зародышей ЖК-фазы, так как порядок во взаимном расположении молекул в этом случае менее строгий. В биологических объектах ЖК-состояние также встречается довольно часто [ $\left.{ }^{2}\right]$. Многими исследователями установлено существование ЖК-фазы для производных целлюлозы - оксипропилцеллюлозы $\left[{ }^{3,4}\right]$ и ацетатов целлюлозы $\left[{ }^{5-9}\right]$. Согласно $\left[{ }^{10}\right]$, сообщения о мезоморфном состоянии немодифицированной целлюлозы строго не обоснованы. Образование ЖК-фазы в производных объясняется тем, что их макромолекулы более жестки и тем самым более склонны к самоупорядочению в растворе, чем молекулы целлюлозы [11]. Исследования данного рода образцов обычно проводили методами поляризационной микроскопии, рассеивания рентгеновского излучения, сканирующей калориметрии, спектрофотометрии, светорассеяния, кругового дихроизма, двойного лучепреломления, вискозиметрии. Только в $\left[{ }^{8}\right]$ наблюдали за расщеплением в спектре ядерного магнитного резонанса протонов хлористого метилена, который является компонентом растворителя. Нанболее интересным и информативным следует считать исследование поведения в мезофазе непосредственно целлюлозы или ее производных как основного вещества, обуславливающего ЖК-упорядочение. ЯМР в различных его вариантах к изучению вышеуказанных объектов до сих пор не привлекался.

В качестве модельной системы нами было исследовано производное глюкозы - октил-1-0- $\beta$ - $D$-глюкозид производства фирмы «Serva» (США). Согласно $\left[{ }^{12,13}\right]$, область реализации ЖК-состояния, определенная методом сканирующей калориметрии, простирается от $67^{\circ}$ до $106^{\circ} \mathrm{C}$. Спектр ЯМР ${ }^{13} \mathrm{C}$ этого соединения в твердом состоянии, снятый при комнатной температуре и при вращении образца под магическим углом $54,7^{\circ}$ для усреднения анизотропных спиновых взаимодействий, приведен на рис. 1, а. Линии гликозидной части расположены в интервале 68-105 м. д., а алифатической - в районе 14-63 м. д. Из спектра следует, что в твердом состоянии реализуется упаковка, при которой соответствующие атомы ${ }^{13} \mathrm{C}$ различных молекул магнитно неэквива- 


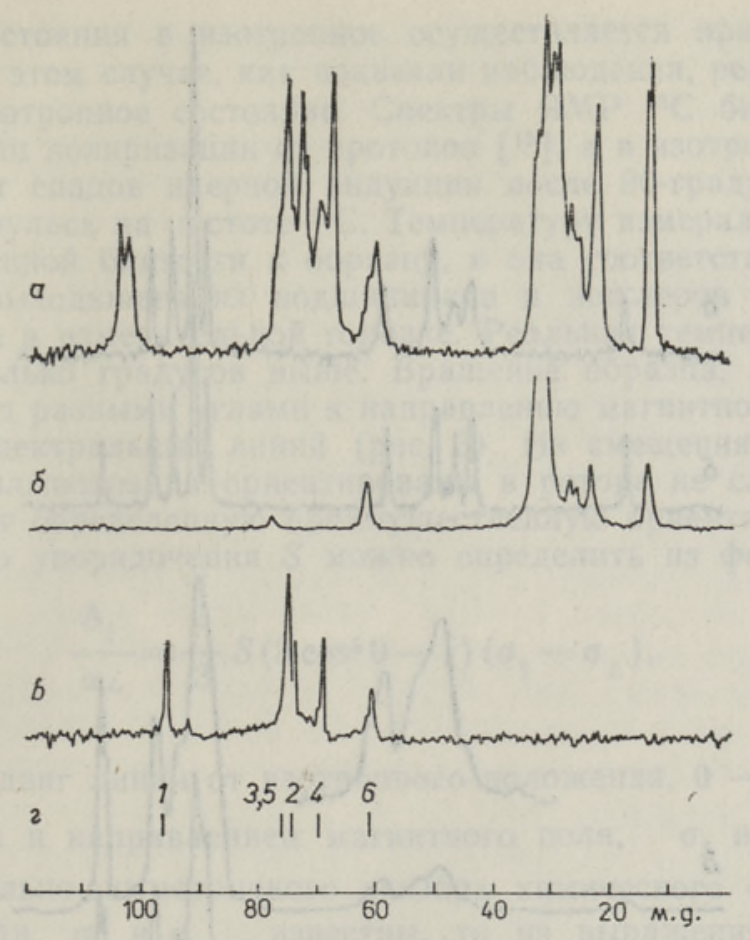

Рис. 1. Спектры ЯМР ${ }^{13} \mathrm{C}$ октил-1-0- $\beta-D$-глюкозида $(a)$, октадецилового спирта (б) и $\beta$ - $D$-глюкозида (в) при температуре $20^{\circ} \mathrm{C}$. Положения линий $\beta$ - $D$-глюкозы в растворенном состоянии (2).

лентны. Хорошо видно расщепление от атомов углерода метильных групп на 14,3 и 15,0 м. д., от атомов углерода С1 - на 103,7 и 105 м. д. Слабые соседние пики имеют почти все линии.

Отнесение спектральных линий проводилось сравнением со спектрами октадецилового спирта $\mathrm{C}_{18} \mathrm{H}_{37} \mathrm{OH}$ (температура плавления $59^{\circ} \mathrm{C}$ ), $\beta-D$-глюкозы в твердом состоянии и в $10 \%$ растворе $\mathrm{H}_{2} \mathrm{O}$ (рис. 1, б-2). Из сравнения следует, что линия при $61-62$ м. д. в спектре октилглюкозида относится к атому С7 алифатической части. Линии при 68,5 и 73,8 м. д. близки к сигналам $\beta$-аномера глюкозы в растворе, химические сдвиги которых составляют 70,2 и 74,8 м. д. Сигнал при 76,2 м. д. следует отнести к двум атомам - С3 и С5, поскольку он совпадает с их общим сигналом в растворе при 76,4 м. Д. Действительно, линия при 76,2 м. д. приблизительно в два раза шире линии от атомов С2 и С4, тем самым ее интегральная интенсивность вдвое больше. Оставшийся сигнал при 70,6 м. д. следует приписать атому С6 глюкозидного звена. Последнее подтверждается тем, что при коротких временах кросс-поляризации относительная интенсивность этого сигнала значительно возрастает, а это, согласно $\left[{ }^{14}\right]$, указывает на то, что он принадлежит атому, у которого больше непосредственно связанных протонов, чем у других.

Для выяснения эффектов ориентации в магнитном поле были сняты спектры октил-1-0- $\beta-D$-глюкозида в ЖК и изотропных состояниях. Спектр образца, быстро вращающегося под магическим углом, при температуре $73^{\circ} \mathrm{C}$ (рис. $\left.2, a\right)$ в области $60-105$ м. д. несколько отличается от спектра твердой фазы (рис. 1,a). Центры тяжести линий на обоих спектрах практически совпадают. Переход в изотропную фазу (рис. 2,6$)$ при повышении температуры детектировался по исчезновению сигнала кросс-поляризации. По нашим измерениям, фазовый пере- 


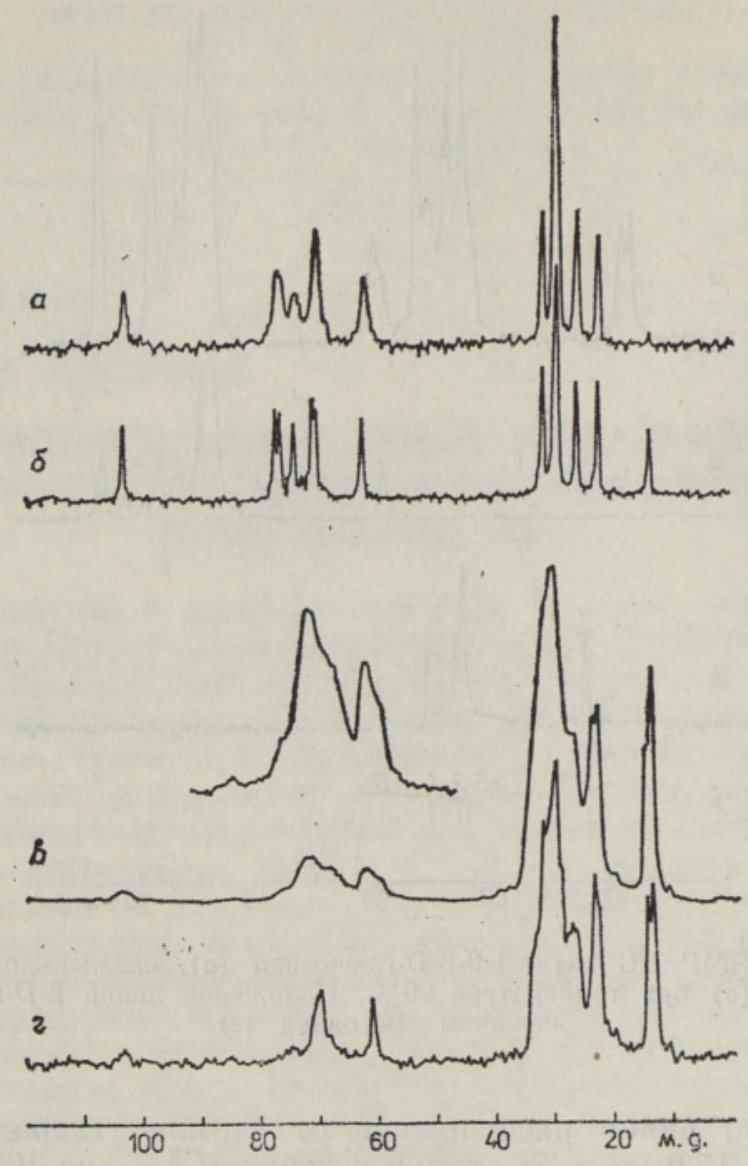

Рис. 2. Спектры ${ }^{13} \mathrm{C}$ октил-1-0- $\beta$ - $D$-глюкознда в жидкокристаллической $(a)$ и изотропной фазе (б). Спектры, полученные без передачи поляризации в твердой фазе при углах вращения $\Theta=50,2^{\circ}$ (в) и $\Theta=54,7^{\circ}$ (2).
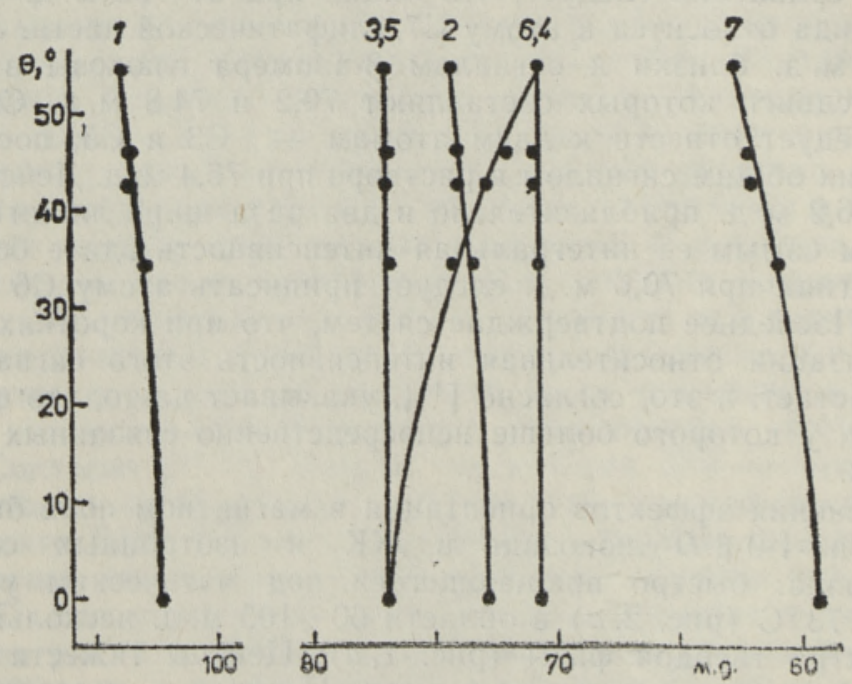

Рнс. 3. Зависимость положения линий в спектре ${ }^{13} \mathrm{C}$ октил-1-0- $\beta-D$-глюкозида от угла вращения образца (в ЖК-фазе). 
ход от ЖК-состояния в изотропное осуществляется при температуре около $90^{\circ} \mathrm{C}$. В этом случае, как показали наблюдения, реализуется как ЖК, так и изотропное состояние. Спектры ЯМР ${ }^{13} \mathrm{C}$ были сняты по методу передачи поляризации от протонов $\left[{ }^{15}\right]$, а в изотропной фаse суммированием спадов ядерной индукции после 90-градусного радиочастотного импульса на частоте ${ }^{13} \mathrm{C}$. Температуру измеряли термопарой в непосредственной близости к образцу, и она соответствовала температуре газа, выходящего из подшипников и жиклеров системы вращения образца в измерительной головке. Реальная температура могла быть на несколько градусов выше. Вращение образца, находящегося в ЖК-фазе, под разными углами к направлению магнитного поля ведет к смещению спектральных линий (рис. 3). Из смещения следует, что молекулы октилглюкозида ориентированы в роторе не случайным образом, а имеют определенную преимущественную ориентацию. Согласно $\left[{ }^{16}\right]$, фактор упорядочения $S$ можно определить из формулы

$$
\frac{\Delta_{\|}}{\omega_{L}}=\frac{1}{3} S\left(3 \cos ^{2} \theta-1\right)\left(\sigma_{\|}-\sigma_{\perp}\right),
$$

где $\frac{\Delta_{\|}}{\omega_{L}}-$ сдвиг линии от изотропного положения, $\theta-$ угол между осью вращения и направлением магнитного поля, $\sigma_{\rrbracket}$ и $\sigma_{\perp}-$ компоненты аксиально-симметричного тензора химического сдвига.

Отсюда, если $\sigma_{\|}$и $\sigma_{\perp}$ известны, то из выражения (1) можно установить преимущественную ориентацию молекул. В $\left[{ }^{17}\right]$ показано, что компоненты тензора химического сдвига можно определить по спектрам двукратного Фурье-преобразования в эксперименте с облучением исследуемого вещества радиочастотными 180-градусными импульсами синхронно с механическим вращением образца. Такой эксперимент требует высокого отношения сигнал/шум в обычном ЯМРспектре (однократного Фурье-преобразования) и усложняется в случае перекрывания линий. Указанные причины не позволили определить параметры химического сдвига. Мы определили эти параметры из спектров образца в твердой фазе при вращении под углом, отличающимся от магического. В этом случае положение сигнала атома зависит от угла вращения и ориентации тензора химического сдвига данного атома следующим образом:

$$
\begin{aligned}
\sigma(\theta, \beta) & =\sigma_{11}\left(\cos ^{2} \theta \sin ^{2} \beta+\frac{1}{2} \sin ^{2} \theta \cos ^{2} \beta\right)+ \\
& +\frac{1}{2} \sigma_{22} \sin ^{2} \theta+\sigma_{33}\left(\cos ^{2} \theta \cos ^{2} \beta+\frac{1}{2} \sin ^{2} \theta \sin ^{2} \beta\right),
\end{aligned}
$$

где $\boldsymbol{\beta}$ - угол между осью механического вращения образца и компонентом $\sigma_{33}$ тензора химического сдвига.

В случае аксиальной симметрии

$$
\begin{aligned}
\sigma(\theta, \beta) & =\sigma_{\perp}\left[\cos ^{2} \theta \sin ^{2} \beta+\frac{1}{2} \sin ^{2} \theta\left(1+\cos ^{2} \beta\right)\right]+ \\
& +\sigma_{\|}\left(\cos ^{2} \theta \cos ^{2} \beta+\frac{1}{2} \sin ^{2} \theta \sin ^{2} \beta\right) .
\end{aligned}
$$

В твердом образце представлены всевозможные ориентации, т. е. $0 \leqslant \beta \leqslant 180^{\circ} ;$ линия размывается в интервале

от $\sigma_{\perp} \sin ^{2} \theta+\sigma_{\|} \cos ^{2} \theta \quad\left(\beta=0,180^{\circ}\right)$ 
до $\sigma_{\perp}\left(\cos ^{2} \theta+\frac{1}{2} \sin ^{2} \theta\right)+\sigma_{\|} \frac{1}{2} \sin ^{2} \theta \quad\left(\beta=90^{\circ}\right)$

и имеет характерное распределение интенсивности. В положении ( $4 a)$ и (4б) наблюдаются сингулярности, где интенсивность резко убывает до нуля. Зная угол вращения $\theta$, нетрудно вычислить $\mid \sigma_{\perp}$ и $\sigma_{\|}$. Аналогичным образом удается определить компоненты $\sigma_{11}, \sigma_{22}$ и $\sigma_{33}$ из сингулярностей в несимметричном случае.

Близкое расположение линий обуславливает перекрывание с удалением от магического угла, поэтому определить сингулярности невозможно. Такие трудности возникают и в нашем случае. Оказывается, что за счет различий во временах релаксации $T_{1 C}$ можно подавить сигналы от атомов C1 до С5. При 1с задержке между накоплениями в спектре, снятом без кросс-поляризации (рис. 2, 2), отчетливо выявляются сигналы от атомов С6 и С7. В спектре, снятом в таком же режиме, но при угле вращения $\theta=50,2^{\circ}$, наблюдается линия, форма.которой характерна для аксиально-симметричного тензора химического сдвига (рис. 2,8$)$. Величина угла $\theta$ была определена из спектра $\mathrm{C}_{6}\left(\mathrm{CH}_{3}\right)_{6}$, снятого при такой же юстировке угла. В расчете использовали значения $\sigma_{\perp}=187,7$ м.д. и $\sigma_{\|}=19,7$ м.д. (ароматические углероды), взятые из литературы $\left[{ }^{18}\right]$. В этом эксперименте важное значение имеет конструкция измерительной головки. В работе применялась измерительная головка с цилиндрическим ротором на воздушных подшипниках, разработанная в Институте химической и биологической физики АН ЭССР, которая позволяет точно и с хорошей воспроизводимостью устанавливать угол вращения $\left[{ }^{19}\right]$. Из сингулярностей спектра с помощью $(4 a)$ и $(4 \sigma)$ для $\sigma_{\perp}$ и $\sigma_{\|}$были получены следующие значения:

$$
\begin{array}{ll}
\sigma_{\perp}=90,6 \text { м. д., } & \sigma_{\|}=29,7 \text { м. д. для атомов C6, } \\
\sigma_{\perp}=74,4 \text { м. д., } & \sigma_{\|}=35,2 \text { м. д. } \text { для атомов С7. }
\end{array}
$$

Сдвиг линии от атомов С7 в сторону сильного поля от изотропного положения при $\theta<54,7^{\circ}$ и $\theta=0^{\circ}$ (неподвижный образец) указывает на тенденцию алифатической части молекулы ориентироваться параллельно магнитному полю. При вращении образца она ориентируется вдоль оси вращения (аналогично нематикам типа МББА и ӘББА). Фактор упо-

рядочения $S$ был определен из отклонения $\frac{\Delta_{\|}}{\omega_{L}}=-3,2$ м.д. при $\theta<0^{\circ}$ и составил 0,12 . Анизотропия химического сдвига атома С6 имеет такой же знак, как и атома С7, что следует из спектра на рис. 2, 2, но линия от С6 смещается в другую сторону. Следовательно, главная ось тензора химического сдвига преимущественно ориентируется перпендикулярно направлению магнитного поля. Расчет по формуле

$$
\frac{\Delta_{\perp}}{\omega_{L}}=\frac{1}{6} S\left(1-3 \cos ^{2} \theta\right)\left(\sigma_{\|}-\sigma_{\perp}\right)
$$

из [ $\left.{ }^{16}\right]$ вместе с $\left.\frac{\Delta_{\perp}}{\omega_{L}}\right|_{\theta=0}=6,4$ м. д. и значениями $\sigma_{\perp}$ и $\sigma_{\|}$ (5б) привел к величине $S=0,32$. Это значение определено в предположении, что главная ось тензора экранирования атома С6 в молекуле октил-1-0- $\beta-D$-глюкозида расположена под углом $90^{\circ}$ к направлению 
преимущественной ориентации всей молекулы. Установить ориентацию и величину компонентов этого тензора одновременно невозможно. Принятое предположение дает минимальные значения фактора упорядочения. Во всех остальных случаях $S>0,32$. Следовательно, в общем случае $S \geqslant 0,32$. Поскольку группа $\mathrm{CH}_{2} \mathrm{OH}$ жестко связана с глюкозидным звеном, приведенное значение характерно и для последнего. Как следует, фактор упорядоченности глюкозидной части молекулы гораздо выше, чем алифатической части. Это указывает на то, что за формнрование ЖК-фазы ответственны в основном глюкозидные части. Снльный сдвиг линии от атома C6 в сторону слабого поля в октил-1-0- $\beta-D$-глюкозиде (по сравнению с $\beta$ - $D$-глюкозидом примерно на $+9,4$ м. д.) указывает на очень сильную водородную связь через $\mathrm{CH}_{2} \mathrm{OH}$-группы с соседней молекулой. Ориентация отдельных фрагментов молекулы согласуется с представлением о реализации в данном веществе смектической фазы за счет водородных связей между глюкозидными звеньями $\left[{ }^{12}\right]$. Соответствующие атомы углерода при этом магнитно-эквивалентны.

Как следует из эксперимента (рис. 3), сигналы от атомов С1 и С2 глюкозидного кольца смещаются гораздо меньше или вообще не смещаются (в случае С $3, \mathrm{C} 4$ и С5), следовательно, они имеют значительно более изотропные тензоры химического сдвига, и тем самым с точки зрения определения ориентации являются менее информативными. Сделанный вывод, по-видимому, распространяется и на полимерные ЖК на основе целлюлозы. Включение атомов С1-C5 в глюкозидное кольцо несколько уменьшает влияние соседних атомов на компоненты тензора химического сдвига, и они остаются малоанизотропными, как было установлено в случае октилглюкозида. Этого нельзя сказать об атоме углерода $\mathrm{CH}_{2} \mathrm{OH}$-группы, имеющей в глюкозе довольно сильную анизотропию и склонной к образованию водородной связи.

При исследовании ЖК на основе целлюлозы особое внимание следует обратить на ориентационные зависимости линии $\mathrm{CH}_{2} \mathrm{OH}$-группы как характеристики основного вещества. Полученные результаты указывают на возможность возникновения характерной для целлюлозы I структуры в жидкокристаллической фазе при синтезе в физиологических условиях.

Авторы выражают благодарность д-ру К. Шуи за предоставление образца октил-1-0- $\beta-D$-глюкозида.

\section{ЛИТЕРАТУРА}

1. Папков С. П. Высокомолекул. соединения. Сер. «А», 26, вып. 5, 1083-1089̊ (1984).

2. Булиаан Н. В кн.: Жидкокристаллический порядок в полимерах (ред. А. Блюмштейн). М., «Мир», 1981, 276-313.

3. Shimamura, K., White, J., Fellers, J. J. Appl. Polym. Sci., 26, № 7, 2165-2180 (1981)

4. Bhadani, S. N., Gray, D. R. Mol. Cryst. Liq. Cryst., 99, № 1, 29-38 (1983).

5. Patel, D. L., Gilbert, R. D. J. Polym. Sci., Polym. Phys. Edn., 19, № 9, 1449-1460 (1981).

6. Lematre, J., Dayan, S., Sixon, P. Mol. Cryst. Liq. Cryst., 84, № 4, 267-273 (1982).

7. Meeten, G. H., Navard, $P$. Polymer, 23, № 12, 1727-1731 (1982).

8. Patel, D. L., Gilbert, R. D. J. Polym. Sci., Polym. Phys. Edn., 20, № 6, 1019-1028 (1982).

9. Юнусов Б. Ю., Ханчич О. А., Серков А. Т., Примкулов М. Т. Высокомолекул. соединения. Сер. «Б», 25, вып. 6, 395-396 (1983).

10. Куличихин В. Г., Голова Л. К. Химия древесины, вып. 3, 9-27 (1985).

11. Папков С. П., Белоусов Ю. Я., Куличихин В. Г. Химические волокна, вып. 3, $8-12(1985)$. 
12. Goodby, J. W. Mol. Cryst. Liq. Cryst., 110, № 1-4, 205-219 (1984).

13. Jeffrey, G. A. Mol. Cryst. Liq. Cryst., 110, № 1-4, 221-237 (1984).

14. Schaefer, J., Stejskal, E. O., Buchdahl, R. Macromolecules, 10, № 2, 384-405 (1972).

15. Pines, A., Gibby, M. G., Waugh, J. S. J. Chem. Phys., 59, № 2, 569-590 (1973).

16. Teeäär, R., Alla, M., Lippmaa, E. Org. Magn. Reson., 19, № 3, 134-137 (1982).

17. Алла М. А., Кундла Э. И., Липпмаа Э. Т. Письма в ЖЭТФ, 27, вып. 4, 208-211 (1978).

18. Pausak, S., Tegenfeldt, J., Waugh, J. S. J. Chem. Phys., 61, № 4, 1338-1344 (1974).

19. Липпмаа Э. Т., Алла М. А., Салумяэ А. А., Тухерм Т. А. Авт. свнд. № 765724. Бюл. изобретений, № 35, 1980.

Институт химической и биологической физики Академии наук Эстонской ССР
Поступила в редакцию 30/III 1987

\section{R. TEEÄAR, E. LIPPMAA}

\section{VEDELA KRISTALLI OKTOUL-1-0- $\beta-D-G L O K O O S I$ ORIENTEERUMISEST MAGNETVÄLJAS}

${ }^{13} \mathrm{C}$-TMR abil on uuritud oktüül-1-0- $\beta$ - $D$-glükoosi molekulide orienteerumist magnetväljas temperatuuridel $20-110^{\circ} \mathrm{C}$. On kindlaks tehtud, et vedela kristalli faasis $\left(67-105^{\circ} \mathrm{C}\right)$ orienteeruvad proovi molekulid oma pikitelgedega eelistatult paralleelselt välise magnetväljaga. Seejuures on glükoosi ringid $\mathrm{CH}_{2} \mathrm{OH}$ rühmade kaudu tugevate vesiniksidemetega seotud, mis põhjustab smektilise faasi kujunemist. Kiirelt pöörlevasse rootorisse paigutatud proovis orienteeruvad molekulid pöörlemise ja välise magnetvälja koosmōjul eelistatult piki proovi pöörlemistelge, kui selle telje ja magnetvälja jöujoonte vaheline nurk on väiksem kui $54,7^{\circ}$. Tahkes faasis on 6 , ja 7 . süsinikuaatomi keemilise nihke tensori komponentideks saadud $\sigma_{\perp}^{(6)}=90,6, \sigma_{\|}^{(6)}=29,7$ ja $\sigma_{\perp}^{(7)}=74,7, \sigma_{\|}^{(7)}=$ $=35,2 \mathrm{ppm}$. Glükoosi ringi süsinikuaatomite keemilise nihke tensorid on tunduvalt isotroopsemad. Saadud väärtuste pōhjal on leitud 6. ja 7. süsinikuaatomi korrastatuse faktori minimaalväärtused, vastavalt 0,32 ja 0,12 . See näitab glükoosi ringide primaarset osa vedela kristalli faasi moodustumisel. Faasiüleminekud tahke ja vedela kristalli ning isotroopse vedeliku faasi vahel on TMR-spektrites selgesti fikseeritavad.

\section{R. TEEĂAR, E. LIPPMAA}

\section{ORIENTATION OF OCTYL-1-0- $\beta-D-$-GLUCOSE IN THE MAGNETIC FIELD AS STUDIED BY NUCLEAR MAGNETIC RESONANCE}

Carbon-13 nuclear magnetic resonance (NMR) spectra of octyl-1-0- $\beta$ - $D$-glucose were measured over a 20 to $110^{\circ} \mathrm{C}$ temperature range. The sample exhibits a liquid crystalline phase between 67 and $105^{\circ} \mathrm{C}$, which causes specific orientation of the molecules. In the liquid crystalline phase placed in a strong magnetic field, the molecules preferably orient in parallel to the field, while in a spinning sample the orientation is in parallel to the spinning axis if the angle between the spinning axis and the magnetic field direction is less than $54.7^{\circ}$. This follows from the NMR line position dependence upon spinning angle. Calculations are based on the values of chemical shift tensor parameters of C-6 and C-7 carbon atoms $\sigma_{\perp}^{(6)}=90.6, \sigma_{\|}^{(6)}=29.7$ and $\sigma_{\perp}^{(7)}=74.4, \sigma_{\|}^{(7)}=35.2 \mathrm{ppm}$, determined from the solid state NMR powder patterns. According to these measurements, the glucose ring carbons have much more isotropic chemical shift tensors. Minimum values of the order parameter were 0.32 and 0.12 corresponding to C-6 and C-7 atoms. This indicates that liquid crystal ordering of the sample is caused mainly by the glucose parts. Phase transitions were clearly seen on the NMR spectra. 